\title{
Critical particle-hole composites at twice the Fermi wave vector in U(1) spin liquid with a Fermi surface
}

\author{
Ki-Seok Kim ${ }^{1,2}$ \\ ${ }^{1}$ Asia Pacific Center for Theoretical Physics, Hogil Kim Memorial building 5th floor, \\ POSTECH, Hyoja-dong, Namgu, Pohang 790-784, Korea \\ ${ }^{2}$ Department of Physics, Pohang University of Science and Technology, Pohang, Gyeongbuk 790-784, Korea
}

(Dated: April 24, 2022)

\begin{abstract}
We find "chiral symmetry breaking" at finite energies in U(1) spin liquid, corresponding to critical particle-hole composite states with twice of the Fermi momentum $\left(2 k_{F}\right)$. We investigate this Fermi surface problem based on the Nambu-Eliashberg theory, where the off diagonal pairing self-energy is introduced to catch the Aslamasov-Larkin vertex correction. This approach is quite parallel with the case of superconductivity, where such Aslamasov-Larkin quantum corrections in the particleparticle channel are well known to be responsible for superconducting instability, formulated as the Nambu-Eliashberg theory in an elegant way. We obtain the pairing self-energy, which vanishes at zero energy but displays the same power law dependence for frequency as the normal Eliashberg selfenergy. As a result, even the pairing self-energy correction does not modify the Eliashberg dynamics without the Nambu spinor representation, where thermodynamics is described by the typical $z=3$ scaling free energy. We discuss physical implication of the anomalous self-energy identical to the conventional Eliashberg normal self-energy, focusing on thermodynamics.
\end{abstract}

PACS numbers: 71.10.-w, 71.10.Hf, 71.27.+a

\section{INTRODUCTION}

Non-Fermi liquid physics in thermodynamics and transport has been at the heart of condensed matter physics. Quantum criticality is regarded as one source of such emergent phenomena ${ }^{1}$, where exotica such as electron fractionalization ${ }^{2}$ and Landau-Ginzburg-Wilson forbidden duality $\underline{3}^{\underline{3}}$ beyond a microscopic model were proposed to explain unique but universal critical phenomena.

An effective field theory for quantum critical dynamics can be generally written in terms of renormalized electrons interacting with order parameter fluctuations ${ }^{4}$, associated with either symmetry breaking or emergent gauge symmetry. Recently, it has been clarified that two dimensional Fermi surface problems are still strongly interacting even in the large- $N$ (spin degeneracy) limit as the non-Abelian gauge theory $\underline{\underline{5-8}} \underline{\underline{-1}}$, where one dimensional processes embedded in two dimensions, i.e., forward and backward scattering near the Fermi surface turn out to be relevant $\stackrel{9}{ }$. One patch formulation was originally examined to keep only the forward scattering, where Landau damping for critical boson dynamics is shown to be exact ${ }^{5}$. Two patch construction was proposed to be a minimal model for the Fermi surface problem, taking into account not only the forward scattering but also the backscattering associated with $2 k_{F}$ particle-hole excitations, where $k_{F}$ is the Fermi momentum. The Landau damping dynamics turns out to be still preserved while the Eliashberg fermion self-energy gets corrected via so called Aslamasov-Larkin (AL) vertex corrections, perturbatively performed to result in an anomalous exponent proportional to $1 / N^{\underline{6}}$. Critical boson dynamics associated with $\mathrm{SU}(2)$ symmetry breaking (ferromagnetism) was considered, arguing that even the dynamical expo- nent may be modified ${ }^{7}$.

Unfortunately, all previous calculations have been performed in a perturbative way based on the Eliashberg solution, thus it is difficult to discuss on non-perturbative critical dynamics of bosons and fermions. Recently, we performed an infinite order summation for particular vertex corrections, given by ladder diagrams 10 . This was performed in a fully self-consistent way, resorting to the Ward identity. It turns out that this class of quantum corrections does not modify the Eliashberg dynamics in the one patch formulation.

AL vertex corrections were perturbatively demonstrated to play an important role in both boson and fermion critical dynamics, as mentioned before. In this respect we need to perform an infinite order summation for this class of quantum corrections. Physically speaking, these quantum processes are possible to cause nontrivial dynamics for $2 k_{F}$ particle-hole excitations. It is well known that the same class of quantum corrections is responsible for superconducting instability of the Fermi liquid state, where a pole with an imaginary frequency in the particle-particle $t$-matrix is identified with a Cooperpair state $\frac{11}{2}$. Similarly, $2 k_{F}$ particle-hole composites may result from AL quantum processes, expected to modify the critical dynamics.

In this paper we propose how to introduce the $\mathrm{AL}$ vertex correction into the two dimensional $\mathrm{U}(1)$ gauge theory with a Fermi surface non-perturbatively. Resorting to the analogy with superconductivity, where the superconducting instability described by the AL vertex correction is reformulated by the anomalous selfenergy in the Eliashberg framework of the Nambu spinor representation 11 , we argue that the off-diagonal selfenergy associated with the $2 k_{F}$ particle-hole channel incorporates the same class of quantum corrections in the 
Nambu spinor representation, where the Nambu spinor is composed of two opposite Fermi momenta, $k_{F}$ and $-k_{F}$. We evaluate an anomalous pairing self-energy in the Nambu-Eliashberg approximation, which vanishes at zero energy but displays the same power law dependence for frequency as the normal Eliashberg selfenergy. As a result, even the pairing self-energy correction does not modify the Eliashberg dynamics without the Nambu spinor representation, where thermodynamics is described by the typical $z=3$ scaling free energy. However, nonzero pairing self-energy corrections at finite frequencies break "chiral symmetry" in the two patch formulation of the $\mathrm{U}(1)$ gauge theory, indicating critical particle-hole composite states with $2 k_{F}$. We discuss physical implication of the anomalous self-energy identical to the conventional Eliashberg normal self-energy, focusing on thermodynamics.

\section{NAMBU-ELIASHBERG THEORY FOR THE} ASLAMASOV-LARKIN VERTEX CORRECTION

\section{A. The ladder vertex correction}

We start from the two patch formulation for the two dimensional U(1) gauge theory with a Fermi surface

$$
\begin{aligned}
& \mathcal{S}_{\text {eff }}=\int_{0}^{\beta} d \tau \int d^{2} r\left\{f_{s \sigma}^{\dagger}\left(\eta \partial_{\tau}-i s \partial_{x}-\partial_{y}^{2}\right) f_{s \sigma}\right. \\
& \left.+\frac{s}{\sqrt{N}} a f_{s \sigma}^{\dagger} f_{s \sigma}+a\left(-\partial_{y}^{2}\right)^{\frac{z-1}{2}} a\right\}
\end{aligned}
$$

where $f_{s \sigma}$ represents a spinon field and $a$ describes $\mathrm{U}(1)$ gauge field in the spin liquid state. $s= \pm$ represents the patch index, corresponding to $+\equiv+k_{F}$ and $-\equiv-k_{F}$, respectively, and $\sigma=1, \ldots, N$ expresses the spin index. Note that the sign in the gauge coupling is different between the two patches, implying the gauge-current minimal coupling. $\eta$ is an infinitesimal coefficient to control artificial divergences in quantum corrections, which can be cured by self-energy corrections ${ }^{5}$. $z$ is the dynamical exponent determining the dispersion relation of gauge fluctuations. It is given by $z=3$ for several problems such as ferromagnetic or nematic quantum criticality including the present spin liquid problem ${ }^{12}$ while $z=2$ in the spin density wave ordering 4 . Both the Fermi velocity $v_{F}$ and the curvature $1 / m$ are set to one. Generally, one may see that this effective field theory describes dynamics of fermions interacting with collective bosons, associated with symmetry breaking.
Dynamics of bosons and fermions can be described selfconsistently by their self-energy corrections. The fermion self-energy is expressed as follows

$$
\begin{aligned}
& \Sigma_{s}\left(k_{0}\right)=-\frac{1}{N} \int \frac{d q_{0}}{2 \pi} \int \frac{d^{2} q}{(2 \pi)^{2}} \Lambda\left(k_{0}+q_{0}, k+q ; k_{0}, k\right) G_{s}\left(k_{0}+q_{0}, k+q\right) D\left(q_{0}, q\right) \\
& -\frac{1}{N} \int \frac{d q_{0}}{2 \pi} \int \frac{d^{2} q}{(2 \pi)^{2}} \Lambda_{2 k_{F}}^{2}\left(k_{0}+q_{0}, k+q ; k_{0}, k\right) G_{-s}\left(k_{0}+q_{0}, k+q\right) D_{2 k_{F}}\left(q_{0}, q\right),
\end{aligned}
$$

where the first part results from the forward scattering associated with $G_{s}\left(k_{0}+q_{0}, k+q\right)$ while the second contribution comes from the backward interaction related with $G_{-s}\left(k_{0}+q_{0}, k+q\right)$, not introduced in the one patch formulation. $G_{s}\left(k_{0}, k\right)$ is the fully renormalized Green's function and $D\left(q_{0}, q\right)$ is the fully renormalized boson propagator, given by

$$
\begin{aligned}
& G_{s}\left(k_{0}, k\right)=\frac{1}{i \eta k_{0}+s k_{x}+k_{y}^{2}-\Sigma_{s}\left(k_{0}\right)}, \\
& D\left(q_{0}, q\right)=\frac{1}{\left|q_{y}\right|^{z-1}+\Pi\left(q_{0}, q\right)},
\end{aligned}
$$

respectively. $\Pi\left(q_{0}, q\right)$ is the boson self-energy, basically given by the fermion polarization function 


$$
\begin{aligned}
& \Pi\left(q_{0}, q\right)=\int \frac{d k_{0}}{2 \pi} \int \frac{d^{2} k}{(2 \pi)^{2}} \Lambda\left(k_{0}+q_{0}, k+q ; k_{0}, k\right) G_{s}\left(k_{0}+q_{0}, k+q\right) G_{s}\left(k_{0}, k\right) \\
& \Pi_{2 k_{F}}\left(q_{0}, q\right)=\int \frac{d k_{0}}{2 \pi} \int \frac{d^{2} k}{(2 \pi)^{2}} \Lambda_{2 k_{F}}^{2}\left(k_{0}+q_{0}, k+q ; k_{0}, k\right) G_{s}\left(k_{0}+q_{0}, k+q\right) G_{-s}\left(k_{0}, k\right)
\end{aligned}
$$

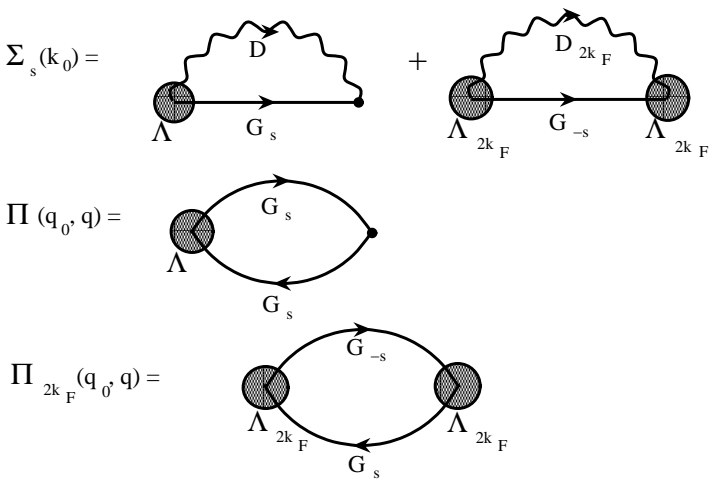

FIG. 1: Fermion self-energy $\left[\Sigma_{s}\left(k_{0}\right)\right]$ and boson self-energies $\left[\Pi\left(q_{0}, q\right)\right.$ and $\left.\Pi_{2 k_{F}}\left(q_{0}, q\right)\right]$ : The fermion self-energy results from both forward and backward scattering and $2 k_{F}$ polarization should be taken into account explicitly in the boson selfenergy. The thick line represents the fermion Green's function and the wavy line does the gauge propagator. The shaded region corresponds to the ladder-type renormalized vertex. where $\Pi_{2 k_{F}}\left(q_{0}, q\right)$ is the fermion polarization function around the $2 k_{F}$ transfer momentum.

$\Lambda\left(k_{0}+q_{0}, k+q ; k_{0}, k\right)$ and $\Lambda_{2 k_{F}}\left(k_{0}+q_{0}, k+q ; k_{0}, k\right)$ are vertex corrections, where the former is associated with the forward scattering and the latter is related with the backscattering, given by

$$
\begin{aligned}
& \Lambda\left(k_{0}+q_{0}, k+q ; k_{0}, k\right)=1 \\
& -\int \frac{d l_{0}}{2 \pi} \int \frac{d^{2} l}{(2 \pi)^{2}} \Lambda\left(k_{0}+q_{0}-l_{0}, k+q-l ; k_{0}-l_{0}, k-l\right) G_{s}\left(k_{0}-l_{0}, k-l\right) G_{s}\left(k_{0}+q_{0}-l_{0}, k+q-l\right) D\left(l_{0}, l\right) \\
& -\int \frac{d l_{0}}{2 \pi} \int \frac{d^{2} l}{(2 \pi)^{2}} \Lambda\left(k_{0}+q_{0}-l_{0}-m_{0}, k+q-l-m ; k_{0}-l_{0}-m_{0}, k-l-m\right) \\
& G_{s}\left(k_{0}-l_{0}-m_{0}, k-l-m\right) G_{s}\left(k_{0}+q_{0}-l_{0}-m_{0}, k+q-l-m\right) D_{2 k_{F}}\left(m_{0}, m\right) \\
& G_{-s}\left(k_{0}-l_{0}, k-l\right) G_{s}\left(k_{0}+q_{0}-l_{0}, k+q-l\right) D_{2 k_{F}}\left(l_{0}, l\right)
\end{aligned}
$$

and

$$
\begin{aligned}
& \Lambda_{2 k_{F}}\left(k_{0}+q_{0}, k+q ; k_{0}, k\right)=1 \\
& -\int \frac{d l_{0}}{2 \pi} \int \frac{d^{2} l}{(2 \pi)^{2}} \Lambda_{2 k_{F}}\left(k_{0}+q_{0}-l_{0}, k+q-l ; k_{0}-l_{0}, k-l\right) G_{-s}\left(k_{0}-l_{0}, k-l\right) G_{s}\left(k_{0}+q_{0}-l_{0}, k+q-l\right) D\left(l_{0}, l\right) \\
& -\int \frac{d l_{0}}{2 \pi} \int \frac{d^{2} l}{(2 \pi)^{2}} \Lambda_{2 k_{F}}\left(k_{0}+q_{0}-l_{0}-m_{0}, k+q-l-m ; k_{0}-l_{0}-m_{0}, k-l-m\right) \\
& G_{-s}\left(k_{0}-l_{0}-m_{0}, k-l-m\right) G_{s}\left(k_{0}+q_{0}-l_{0}-m_{0}, k+q-l-m\right) D_{2 k_{F}}\left(m_{0}, m\right) \\
& G_{s}\left(k_{0}-l_{0}, k-l\right) G_{s}\left(k_{0}+q_{0}-l_{0}, k+q-l\right) D_{2 k_{F}}\left(l_{0}, l\right)
\end{aligned}
$$

respectively, in the ladder approximation. It is straightforward to read these vertex corrections. The first part in the forward scattering vertex is that $G_{s}\left(k_{0}, k\right)$ emits
$D\left(l_{0}, l\right)$ to be $G_{s}\left(k_{0}-l_{0}, k-l\right)$ and scatters into $G_{s}\left(k_{0}+\right.$ $\left.q_{0}-l_{0}, k+q+l\right)$ by $\Lambda\left(k_{0}+q_{0}-l_{0}, k+q-l ; k_{0}-l_{0}, k-l\right)$, accepting $D\left(l_{0}, l\right)$ to be $G_{s}\left(k_{0}+q_{0}, k+q\right)$. In the second cor- 


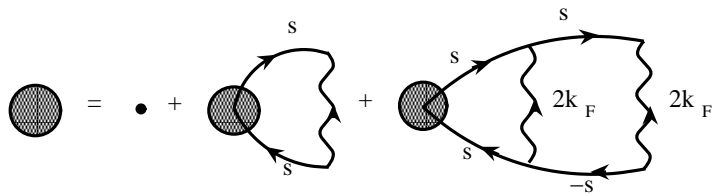

FIG. 2: (Color online) The ladder vertex correction near the zero momentum transfer turns out to be irrelevant in the Eliashberg solution.

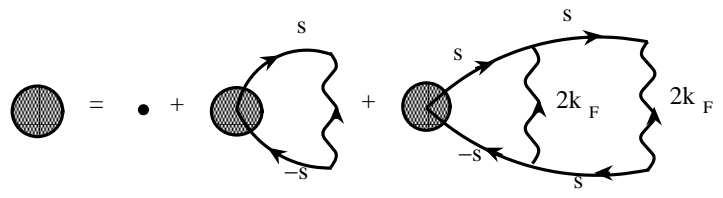

FIG. 3: (Color online) The ladder vertex correction near the $2 k_{F}$ transfer momentum turns out to be singular in the Eliashberg solution, and should be taken into account carefully.

rection $G_{s}\left(k_{0}, k\right)$ emits $D_{2 k_{F}}\left(l_{0}, l\right)$ to be $G_{-s}\left(k_{0}-l_{0}, k-l\right)$, which scatters into $G_{s}\left(k_{0}-l_{0}-m_{0}, k-l-m\right)$ by $D_{2 k_{F}}\left(m_{0}, m\right) . \quad G_{s}\left(k_{0}-l_{0}-m_{0}, k-l-m\right)$ becomes $G_{s}\left(k_{0}+q_{0}-l_{0}-m_{0}, k+q-l-m\right)$ by $\Lambda\left(k_{0}+q_{0}-\right.$ $\left.l_{0}-m_{0}, k+q-l-m ; k_{0}-l_{0}-m_{0}, k-l-m\right)$, and it accepts $D_{2 k_{F}}\left(m_{0}, m\right)$ to be $G_{s}\left(k_{0}+q_{0}-l_{0}, k+q-l\right)$. It again accepts $D_{2 k_{F}}\left(l_{0}, l\right)$ to be $G_{s}\left(k_{0}+q_{0}, k+q\right)$. One can understand the $2 k_{F}$ vertex correction in the same way. Equations (2), (3), (4), (5), and (6) consist of a fully selfconsistent framework for a particular class of quantum corrections in the Fermi surface problem.

If the backward scattering given by $\Lambda_{2 k_{F}}\left(k_{0}+q_{0}, k+\right.$ $\left.q ; k_{0}, k\right)$ is neglected, these self-consistent equations are reduced to those of the one patch formulation. As mentioned in the introduction, such forward scattering contributions do not modify the Eliashberg dynamics ${ }^{10}$. The ladder vertex correction was solved, resorting to the Ward identity with an ansatz, where the vertex function is given by the fully renormalized Green's function. Such an ansatz turns out to recover both the non-interacting limit and one dimensional case. Inserting this ansatz expression into both self-energy equations, we obtain fully self-consistent solutions, basically the same as the Eliashberg self-energies in the low energy limit.

However, the role of the backscattering was emphasized because the $2 k_{F}$ ladder vertex turns out to be singular ${ }^{13}$, given by

$$
\Lambda_{2 k_{F}}\left(k_{0}+q_{0}, k+q ; k_{0}, k\right)=\frac{\Lambda_{0}}{\left\{\left|\frac{q_{0}}{\epsilon_{F}}\right|+c\left(\frac{\sqrt{q_{x}^{2}+q_{y}^{2}}}{2 k_{F}}\right)^{\frac{3}{2}}\right\}^{\eta}},(7)
$$

where the transfer momentum $q$ is already expanded near $2 k_{F}$. $\epsilon_{F}$ is the Fermi energy and $c$ is a positive numerical constant of the order of one. $\Lambda_{0}$ is the bare vertex, set to be 1 in this paper. It should be addressed that this singular $2 k_{F}$ particle-hole ladder vertex was not obtained selfconsistently. Instead, it was found from a perturbative summation up to an infinite order (parquet approxima- tion), based on the Eliashberg Green's function ${ }^{13}$. Unfortunately, we do not know how to get a self-consistent vertex solution, where the Ward identity cannot be used for the $2 k_{F}$ channel. In this subsection we discuss several possibilities based on the previous perturbative vertex solution.

$\eta$ is an anomalous exponent for the $2 k_{F}$ vertex function, depending on the spin degeneracy and given by $1 / N$ basically. Inserting this vertex into Eq. (4), the previous analysis found two kinds of boson self-energy corrections 13

$$
\begin{aligned}
& \Pi_{2 k_{F}}\left(q_{0}, q ; \eta<\eta_{c}\right) \\
& =\mathcal{P}-\sqrt{\frac{p_{0}}{\omega_{0} v_{F}^{3}}}\left\{c_{\omega}\left(\frac{q_{0}}{\omega_{0}}\right)^{\frac{2}{3}-2 \eta}+c_{q}\left|\frac{\sqrt{q_{x}^{2}+q_{y}^{2}}}{2 k_{F}}\right|^{1-3 \eta}\right\}, \\
& \Pi_{2 k_{F}}\left(q_{0}, q ; \eta>\eta_{c}\right) \\
& =\sqrt{\frac{p_{0}}{\omega_{0} v_{F}^{3}}}\left\{c_{\omega}\left(\frac{q_{0}}{\omega_{0}}\right)^{2 \eta-\frac{2}{3}}+c_{q}\left|\frac{\sqrt{q_{x}^{2}+q_{y}^{2}}}{2 k_{F}}\right|^{3 \eta-1}\right\}^{-1},(8)
\end{aligned}
$$

where the critical value is $\eta_{c}=1 / 3 . \omega_{0}=\left(\frac{1}{2 \sqrt{3}}\right)^{3} \frac{2 v_{F}^{3} g^{4}}{\pi^{2} p_{0}}$, where $v_{F}$ and $p_{0}$ are the Fermi velocity and curvature, respectively, and $g$ is the gauge coupling constant. Remember $v_{F}, p_{0}, g=1$ in this paper.

The $2 k_{F}$ boson self-energy does not diverge in $\eta<\eta_{c}$ and can be neglected at low energies due to a positive constant $\mathcal{P}$. On the other hand, it becomes singular when $\eta$ exceeds $\eta_{c}$, implying quantum criticality of the boson state. An important question is how the fermion self-energy behaves, scattered by this $2 k_{F}$ critical boson dynamics. Does it diverge to give rise to an instability to a density wave (charge or spin) or vanish with an anomalous exponent? The latter will be identified with a quantum critical state, characterized by a diverging $2 k_{F}$ spin susceptibility. We believe that this question is not addressed appropriately, i.e., fully selfconsistently even in the relativistic $\mathrm{U}(1)$ gauge theory, where the $1 / N$ expansion is well defined. It was demonstrated that the ladder vertex correction results in singularity to the staggered spin susceptibility, based on the $1 / N$ expansion in the relativistic $\mathrm{U}(1)$ gauge theory ${ }^{14}$. Since the fermion self-energy vanishes with an anomalous critical exponent, the quantum criticality characterized by the divergent staggered spin susceptibility can be regarded as a stable phase, sometimes identified with an algebraic spin liquid as one possible phase of the paramagnetic Mott insulator ${ }^{2}$. This statement is certainly plausible at $N=N_{c}$, below which chiral symmetry breaking arises to result in an antiferromagnetic order, because $N=N_{c}$ corresponds to an antiferromagnetic quantum critical point 15 . Our real question is whether this critical solution survives or not away from the quantum critical point, i.e., in the case when $N>N_{c}$. Although the previous non-self-consistent analysis demonstrated quantum criticality of the antiferromagnetic spin susceptibility in the algebraic spin liquid state $\left(N>N_{c}\right)$, it seems to be possible that a fully self-consistent treatment may cause 
both the fermion self-energy and the staggered spin susceptibility to vanish with non-trivial critical exponents.

The present Fermi surface problem is much more complicated because the $1 / N$ expansion is not well defined ${ }^{5}$. Inserting the boson self-energy of Eq. (8) into Eq. (2), one can find the fermion self-energy, particulary corrected by the $2 k_{F}$ process. As discussed above, it is not clear whether $\eta$ exceeds its critical value or not. When $\eta$ is smaller than $\eta_{c}$, the $2 k_{F}$ scattering is irrelevant due to gapped boson excitations at this momentum, and the Eliashberg dynamics will be preserved. However, the divergent boson self-energy in the case of $\eta>\eta_{c}$ makes the fermion self-energy more singular than the Eliashberg one. Actually, inserting the singular $2 k_{F}$ ladder vertex and the divergent $2 k_{F}$ boson self-energy into the equation for the fermion self-energy, we obtain

$$
\Sigma_{2 k_{F}}\left(k_{0} ; \eta>\eta_{c}\right) \propto-i \frac{\operatorname{sgn}\left(k_{0}\right)}{N}\left|k_{0}\right|^{\frac{1}{3}-2 \eta},
$$

which diverges in the $k_{0} \rightarrow 0$ limit. This can be interpreted as a particle-hole bound state, analogous with a Cooper pair in the particle-particle channel, resulting in a density wave state, where gauge fluctuations play the role of a pairing glue, reflected in their divergent self-energy.

We believe that this $2 k_{F}$ issue should be addressed self-consistently in both relativistic and non-relativistic $\mathrm{U}(1)$ gauge theories. One way to resolve this issue is discussed in the last subsection. In this paper we assume that the $2 k_{F}$ ladder process does not cause any change to the Eliashberg dynamics, i.e., $\eta<\eta_{c}$, and investigate other quantum corrections.

\section{B. Review on the Aslamasov-Larkin vertex correction associated with the superconducting instability}

We review the role of AL quantum corrections in the Eliashberg dynamics, demonstrated to cause an anomalous exponent beyond the Eliashberg fermion self-energy. As discussed in the introduction, such AL vertex corrections in the particle-particle channel were shown to cause superconducting instability to the Fermi liquid state ${ }^{11}$. Interestingly, this $t$-matrix construction was reformulated in the particle-hole Nambu representation, resulting in the same critical temperature. In this subsection we focus on the connection between the Nambu-Eliashberg theory and the AL quantum corrected self-energy.

The electron self-energy is given by

$$
\Sigma\left(p_{0}\right)=-\int \frac{d q_{0}}{2 \pi} \int \frac{d^{3} q}{(2 \pi)^{3}} \Gamma\left(p_{0}+q_{0}, p+q ; p_{0}, p\right) G\left(p_{0}+q_{0}, p+q\right) g_{q} D\left(q_{0}, q\right)
$$

where $g_{q}$ is the electron-phonon coupling constant and $\Gamma\left(p_{0}+q_{0}, p+q ; p_{0}, p\right)$ is the associated vertex. $D\left(q_{0}, q\right)$ is the phonon propagator. Here, the imaginary time for- mulation is applied at zero temperature. The electronphonon vertex can be approximated as follows (Fig. 4)

$$
\begin{aligned}
\Gamma\left(p_{0}+q_{0}, p+q ; p_{0}, p\right) & =g_{q} \\
& -g_{q} \int \frac{d k_{0}}{2 \pi} \int \frac{d^{3} k}{(2 \pi)^{3}} \mathbf{T}_{p p}\left[\left(k_{0}, k ; p_{0}+q_{0}, p+q\right)\left(k_{0}+q_{0}, k+q ; p_{0}, p\right)\right] G\left(k_{0}+q_{0}, k+q\right) G\left(k_{0}, k\right)
\end{aligned}
$$

where $\mathbf{T}_{p p}\left[\left(k_{0}, k ; p_{0}+q_{0}, p+q\right)\left(k_{0}+q_{0}, k+q ; p_{0}, p\right)\right]$ is the particle-particle $t$-matrix to show the transition process that $\left(p_{0}, p\right)$ and $\left(k_{0}+q_{0}, k+q\right)$ scatter into $\left(p_{0}+q_{0}, p+q\right)$ and $\left(k_{0}, k\right)$ with the transfer momentum $\left(q_{0}, q\right)$, respectively. Inserting this AL vertex into the fermion selfenergy, we obtain 


$$
\begin{aligned}
& \Sigma\left(p_{0}\right) \approx-\int \frac{d q_{0}}{2 \pi} \int \frac{d^{3} q}{(2 \pi)^{3}} G\left(p_{0}+q_{0}, p+q\right) g_{q} D\left(q_{0}, q\right) g_{q} \\
& +\int \frac{d q_{0}}{2 \pi} \int \frac{d^{3} q}{(2 \pi)^{3}} \mathbf{T}_{p p}\left[\left(-p_{0},-p ; p_{0}+q_{0}, p+q\right)\left(-p_{0}+q_{0},-p+q ; p_{0}, p\right)\right] \\
& G\left(p_{0}+q_{0}, p+q\right) G\left(-p_{0},-p\right) G\left(-p_{0}+q_{0},-p+q\right) g_{q} D\left(q_{0}, q\right) g_{q}
\end{aligned}
$$

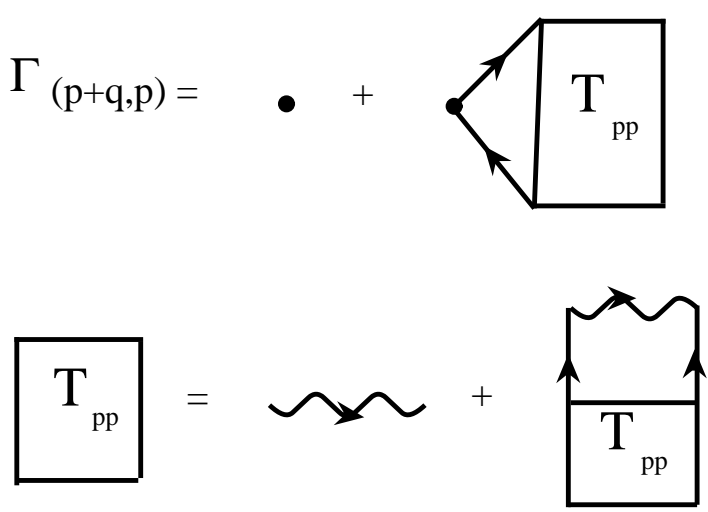

FIG. 4: (Color online) The $\mathrm{AL}$ vertex correction in the particle-particle channel for superconducting instability

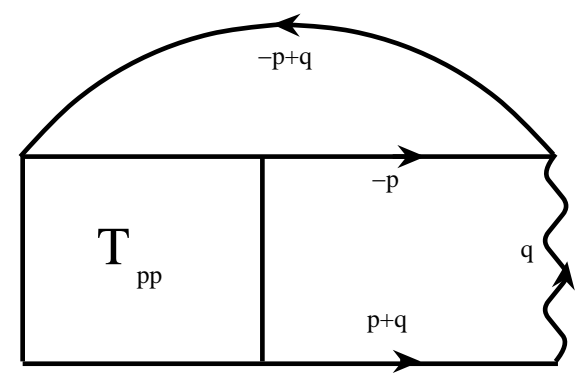

FIG. 5: (Color online) The electron self-energy by the AL vertex correction in the particle-particle channel for superconducting instability

The particle-particle $t$-matrix is given by the BetheSalpeter equation 11

where the most singular particle-particle channel was selected (Fig. 5).

$$
\begin{aligned}
& \mathbf{T}_{p p}\left[\left(-p_{0},-p ; p_{0}+q_{0}, p+q\right)\left(-p_{0}+q_{0},-p+q ; p_{0}, p\right)\right]=g_{q} D\left(q_{0}, q\right) g_{q} \\
& +\int \frac{d k_{0}^{\prime}}{2 \pi} \int \frac{d^{3} k^{\prime}}{(2 \pi)^{3}} \mathbf{T}_{p p}\left[\left(-p_{0}+q_{0}-k_{0}^{\prime},-p+q-k^{\prime} ; p_{0}+k_{0}^{\prime}, p+k^{\prime}\right)\left(-p_{0}+q_{0},-p+q ; p_{0}, p\right)\right] \\
& G\left(p_{0}+k_{0}^{\prime}, p+k^{\prime}\right) G\left(-p_{0}+q_{0}-k_{0}^{\prime},-p+q-k^{\prime}\right) g_{q-k^{\prime}} D\left(q_{0}-k_{0}^{\prime}, q-k^{\prime}\right) g_{q-k^{\prime}} .
\end{aligned}
$$

See Fig. 4. Generally speaking, this integral equation cannot be solved analytically because it is not factorized. But, if the retardation effect can be neglected, one obtains its analytic expression ${ }^{11}$.

We compare this approach with an elegant reformulation based on the Nambu-Eliashberg approximation. The
Dyson equation is

$$
\boldsymbol{G}\left(p_{0}, p\right)=\boldsymbol{g}\left(p_{0}, p\right)+\boldsymbol{g}\left(p_{0}, p\right) \boldsymbol{\Sigma}\left(p_{0}\right) \boldsymbol{G}\left(p_{0}, p\right),
$$

where the Green's function is a two by two matrix in the 
Nambu spinor representation, given by

$$
\begin{aligned}
& \boldsymbol{G}\left(p_{0}, p\right) \equiv-\left\langle\left(\begin{array}{c}
c_{p \uparrow} \\
c_{-p \downarrow}^{\dagger}
\end{array}\right)\left(\begin{array}{cc}
c_{p \uparrow}^{\dagger} & c_{-p \downarrow}
\end{array}\right)\right\rangle \\
& \equiv\left(\begin{array}{cc}
G\left(p_{0}, p\right) & F\left(p_{0}, p\right) \\
F^{*}\left(p_{0}, p\right) & -G\left(-p_{0},-p\right)
\end{array}\right) .
\end{aligned}
$$

$G\left(p_{0}, p\right)$ is a normal Green's function with a bare propagator $g\left(p_{0}, p\right)$ while $F\left(p_{0}, p\right)$ is an anomalous propagator associated with particle-particle pairing. The electron self-energy is given by

$$
\boldsymbol{\Sigma}\left(p_{0}\right)=\left(\begin{array}{cc}
\Sigma\left(p_{0}\right) & \Phi\left(p_{0}\right) \\
\Phi^{*}\left(p_{0}\right) & -\Sigma\left(-p_{0}\right)
\end{array}\right)
$$

where $\Phi\left(p_{0}\right)$ is the pairing self-energy and $\Sigma\left(p_{0}\right)$, the normal one. Then, the Dyson equation becomes

$$
\begin{aligned}
& G\left(p_{0}, p\right)=g\left(p_{0}, p\right)+g\left(p_{0}, p\right) \Sigma\left(k_{0}\right) G\left(p_{0}, p\right)+g\left(p_{0}, p\right) \Phi\left(p_{0}\right) F^{*}\left(p_{0}, p\right), \\
& F^{*}\left(p_{0}, p\right)=-g\left(-p_{0},-p\right) \Phi^{*}\left(p_{0}\right) G\left(p_{0}, p\right)+g\left(-p_{0},-p\right) \Sigma\left(-p_{0}\right) F^{*}\left(p_{0}, p\right)
\end{aligned}
$$

Inserting

$$
F^{*}\left(p_{0}, p\right)=-\frac{g\left(-p_{0},-p\right) \Phi^{*}\left(p_{0}\right) G\left(p_{0}, p\right)}{1-g\left(-p_{0},-p\right) \Sigma\left(-p_{0}\right)}
$$

from the second equation into the first, we obtain the full renormalized self-energy in the Nambu formalism

$$
\Sigma_{t o t}\left(p_{0}\right)=\Sigma\left(p_{0}\right)-\frac{g\left(-p_{0},-p\right)\left|\Phi\left(p_{0}\right)\right|^{2}}{1-g\left(-p_{0},-p\right) \Sigma\left(-p_{0}\right)},
$$

where the total self-energy is defined as

$$
G\left(p_{0}, p\right) \equiv g\left(p_{0}, p\right)+g\left(p_{0}, p\right) \Sigma_{t o t}\left(p_{0}\right) G\left(p_{0}, p\right) .
$$

The self-energy is determined in the Eliashberg approximation

$$
\boldsymbol{\Sigma}\left(p_{0}\right)=\int \frac{d q_{0}}{2 \pi} \int \frac{d^{3} q}{(2 \pi)^{3}} g_{p h}^{2} D\left(q_{0}, q\right) \boldsymbol{G}\left(p_{0}+q_{0}, p+q\right)
$$

Inserting the Nambu Green's function into the above expression, we find full self-consistent equations

$$
\begin{aligned}
& \Phi^{*}\left(p_{0}\right)=-\int \frac{d q_{0}}{2 \pi} \int \frac{d^{3} q}{(2 \pi)^{3}} g_{p h}^{2} D\left(q_{0}, q\right) \frac{g\left(-p_{0}-q_{0},-p-q\right) \Phi^{*}\left(p_{0}+q_{0}\right)}{1-g\left(-p_{0}-q_{0},-p-q\right) \Sigma\left(-p_{0}-q_{0}\right)} \\
& \frac{g\left(p_{0}+q_{0}, p+q\right)}{1-g\left(p_{0}+q_{0}, p+q\right)\left\{\Sigma\left(p_{0}+q_{0}\right)-\frac{g\left(-p_{0}-q_{0},-p-q\right)\left|\Phi\left(p_{0}+q_{0}\right)\right|^{2}}{1-g\left(-p_{0}-q_{0},-p-q\right) \Sigma\left(-p_{0}-q_{0}\right)}\right\}}
\end{aligned}
$$

for the pairing self-energy and

$$
\Sigma\left(p_{0}\right)=\int \frac{d q_{0}}{2 \pi} \int \frac{d^{3} q}{(2 \pi)^{3}} g_{p h}^{2} D\left(q_{0}, q\right) \frac{g\left(p_{0}+q_{0}, p+q\right)}{1-g\left(p_{0}+q_{0}, p+q\right)\left\{\Sigma\left(p_{0}+q_{0}\right)-\frac{g\left(-p_{0}-q_{0},-p-q\right)\left|\Phi\left(p_{0}+q_{0}\right)\right|^{2}}{1-g\left(-p_{0}-q_{0},-p-q\right) \Sigma\left(-p_{0}-q_{0}\right)}\right\}}
$$

for the normal electron self-energy.

In order to see similarity between the $t$-matrix approx- imation and the Nambu-Eliashberg one, we perform the following approximation for the normal self-energy

$$
\begin{aligned}
& \Sigma\left(p_{0}\right) \approx \int \frac{d q_{0}}{2 \pi} \int \frac{d^{3} q}{(2 \pi)^{3}} g\left(p_{0}+q_{0}, p+q\right) g_{p h} D\left(q_{0}, q\right) g_{p h} \\
& -\int \frac{d q_{0}}{2 \pi} \int \frac{d^{3} q}{(2 \pi)^{3}}\left|\Phi\left(p_{0}+q_{0}\right)\right|^{2} g\left(p_{0}+q_{0}, p+q\right) g\left(p_{0}+q_{0}, p+q\right) g\left(-p_{0}-q_{0},-p-q\right) g_{p h} D\left(q_{0}, q\right) g_{p h}
\end{aligned}
$$


Comparing this expression with Eq. (12) in the $t$-matrix self-energy, we find some similarity if we identify the $t$ - matrix [Eq. (13)] with the square of the pairing selfenergy $[\mathrm{Eq} .(17)]$ in the following approximation

$$
\Phi^{*}\left(p_{0}\right) \approx-\int \frac{d q_{0}}{2 \pi} \int \frac{d^{3} q}{(2 \pi)^{3}} g_{p h}^{2} D\left(q_{0}, q\right) g\left(p_{0}+q_{0}, p+q\right) g\left(-p_{0}-q_{0},-p-q\right) \Phi^{*}\left(p_{0}+q_{0}\right) .
$$

It is clear that the $t$-matrix approximation is not equivalent to the Nambu-Eliashberg formulation in the normal state because the anomalous pairing self-energy should vanish as discussed in the next section. However, we believe that the anomalous self-energy in the NambuEliashberg theory will catch an essential physics of the AL vertex-corrected electron self-energy at the critical point, where the singular part of the normal self-energy results from the pairing one in the Nambu-Eliashberg approximation while from the $t$-matrix process in the $\mathrm{AL}$ vertex correction. One can regard the Nambu representation as one possible way to select a better basis set for an instability channel via "unitary transformation".

\section{Nambu-Eliashberg theory}

One can construct a self-consistent framework for boson and fermion self-energy corrections, introducing the $\mathrm{AL}$ vertex with the $2 k_{F}$ particle-hole $t$-matrix BetheSalpeter equation. However, this strategy is not practical because such self-consistent equations are much compli- cated, where the retardation effect is inevitable. As the $\mathrm{AL}$ vertex correction is reformulated based on the Nambu spinor representation in superconductivity, we introduce such quantum processes in the same way, but the component of the Nambu spinor will be different from that in superconductivity.

Resorting to the analogy with superconductivity, we rewrite the two patch model Eq. (1) in the Nambu spinor representation

$$
\begin{aligned}
& \mathcal{S}_{\text {eff }}=\int_{0}^{\beta} d \tau \int d^{2} r\left\{F_{\sigma}^{\dagger}\left(\eta \partial_{\tau} \boldsymbol{I}-i \partial_{x} \boldsymbol{\tau}_{\mathbf{3}}-\partial_{y}^{2} \boldsymbol{I}\right) F_{\sigma}\right. \\
& \left.+\frac{1}{\sqrt{N}} a F_{\sigma}^{\dagger} \boldsymbol{\tau}_{\mathbf{3}} F_{\sigma}+a\left(-\partial_{y}^{2}\right)^{\frac{z-1}{2}} a\right\}
\end{aligned}
$$

where $F_{\sigma}=\left(\begin{array}{c}f_{+\sigma} \\ f_{-\sigma}\end{array}\right)$ is the Nambu spinor composed of each-patch fermion. $\tau_{\mathbf{3}}$ acts on the $+k_{F}$ particle and $-k_{F}$ particle space.

Both boson and fermion self-energies can be found in the Nambu-Eliashberg theory

$$
\begin{aligned}
& \Pi\left(q_{0}, q\right)=\boldsymbol{t} \boldsymbol{r} \int \frac{d k_{0}}{2 \pi} \int \frac{d^{2} k}{(2 \pi)^{2}} \boldsymbol{\tau}_{\mathbf{3}} \boldsymbol{G}\left(k_{0}+q_{0}, k+q\right) \boldsymbol{\tau}_{\mathbf{3}} \boldsymbol{G}\left(k_{0}, k\right), \\
& \boldsymbol{\Sigma}\left(k_{0}\right)=-\frac{1}{N} \int \frac{d q_{0}}{2 \pi} \int \frac{d^{2} q}{(2 \pi)^{2}} \boldsymbol{\tau}_{\mathbf{3}} \boldsymbol{G}\left(k_{0}+q_{0}, k+q\right) D\left(q_{0}, q\right),
\end{aligned}
$$

where

$$
\boldsymbol{G}\left(k_{0}, k\right)=\left(i \eta k_{0} \boldsymbol{I}+k_{x} \boldsymbol{\tau}_{\mathbf{3}}+k_{y}^{2} \boldsymbol{I}-\boldsymbol{\Sigma}\left(k_{0}\right)\right)^{-1}
$$

is the fermion Nambu Green's function with the matrix self-energy

$$
\boldsymbol{\Sigma}\left(k_{0}\right)=\left(\begin{array}{cc}
\Sigma\left(k_{0}\right) & \Phi\left(k_{0}\right) \\
\Phi^{*}\left(k_{0}\right) & \Sigma\left(k_{0}\right)
\end{array}\right)
$$

$\Sigma\left(k_{0}\right)$ is the normal part and $\Phi\left(k_{0}\right)$ is an effective pairing potential. More explicitly, the normal fermion selfenergy, the fermion pairing potential, and the boson selfenergy are given by 


$$
\begin{aligned}
\Sigma\left(k_{0}\right) & =-\frac{1}{N} \int \frac{d q_{0}}{2 \pi} \int \frac{d^{2} q}{(2 \pi)^{2}} G\left(k_{0}+q_{0}, k+q\right) D\left(q_{0}, q\right), \\
\Phi\left(k_{0}\right) & =\frac{1}{N} \int \frac{d q_{0}}{2 \pi} \int \frac{d^{2} q}{(2 \pi)^{2}} F\left(k_{0}+q_{0}, k+q\right) D\left(q_{0}, q\right), \\
\Pi\left(q_{0}, q\right) & =\int \frac{d k_{0}}{2 \pi} \int \frac{d^{2} k}{(2 \pi)^{2}}\left\{G\left(k_{0}+q_{0}, k+q\right) G\left(k_{0}, k\right)+G\left(k_{0}+q_{0},-k-q\right) G\left(k_{0},-k\right)\right. \\
& \left.-F\left(k_{0}+q_{0}, k+q\right) F^{*}\left(k_{0}, k\right)-\text { H.c. }\right\},
\end{aligned}
$$

where

$$
\begin{aligned}
G\left(k_{0}, k\right) & =\frac{i \eta k_{0}-k_{x}+k_{y}^{2}-\Sigma\left(k_{0}\right)}{\left(i \eta k_{0}+k_{y}^{2}-\Sigma\left(k_{0}\right)\right)^{2}-k_{x}^{2}-\left|\Phi\left(k_{0}\right)\right|^{2}}, \\
F\left(k_{0}, k\right) & =-\frac{\Phi\left(k_{0}\right)}{\left(i \eta k_{0}+k_{y}^{2}-\Sigma\left(k_{0}\right)\right)^{2}-k_{x}^{2}-\left|\Phi\left(k_{0}\right)\right|^{2}}
\end{aligned}
$$

are normal and abnormal fermion propagators, respectively.

\section{A solution of the Eliashberg equation}

Although it is not easy to solve Eq. (23) in a general way, the Landau damping ansatz for the boson selfenergy

$$
\Pi\left(q_{0}, q\right)=\gamma \frac{\left|q_{0}\right|}{\left|q_{y}\right|}
$$

makes this problem straightforward. In appendix we will check that the Landau damping boson self-energy is selfconsistent indeed.
Inserting the Landau damping boson self-energy into the boson propagator, normal and anomalous fermion self-energies satisfy

$$
\begin{aligned}
& \Sigma\left(k_{0}\right)=\frac{i}{2 N}\left\{\int_{0}^{\infty} \frac{d y}{\pi} \frac{y}{y^{z}+1}\right\} \int_{-k_{0}}^{0} \frac{d q_{0}}{2 \pi}\left(\gamma\left|q_{0}\right|\right)^{\frac{2-z}{z}} \frac{\Sigma\left(k_{0}+q_{0}\right)}{\sqrt{\Sigma^{2}\left(k_{0}+q_{0}\right)-\left|\Phi\left(k_{0}+q_{0}\right)\right|^{2}}} \\
& \Phi\left(k_{0}\right)=\frac{i}{2 N}\left\{\int_{0}^{\infty} \frac{d y}{\pi} \frac{y}{y^{z}+1}\right\} \int_{-k_{0}}^{0} \frac{d q_{0}}{2 \pi}\left(\gamma\left|q_{0}\right|\right)^{\frac{2-z}{z}} \frac{\Phi\left(k_{0}+q_{0}\right)}{\sqrt{\Sigma^{2}\left(k_{0}+q_{0}\right)-\left|\Phi\left(k_{0}+q_{0}\right)\right|^{2}}}
\end{aligned}
$$

These self-consistent equations are typical in the NambuEliashberg theory.

Considering the symmetry $\Sigma\left(k_{0}\right) \longleftrightarrow \Phi\left(k_{0}\right)$ in Eq. (26), we propose

$$
\begin{aligned}
& \Sigma\left(k_{0}\right)=-i \frac{\lambda}{N} \operatorname{sgn}\left(k_{0}\right)\left|k_{0}\right|^{\frac{2}{z}}, \\
& \Phi\left(k_{0}\right)=-i \frac{\chi}{N} \operatorname{sgn}\left(k_{0}\right)\left|k_{0}\right|^{\frac{2}{z}}
\end{aligned}
$$

as a possible solution. Inserting this expression into Eq. (26), we see 


$$
\begin{aligned}
& \Sigma\left(k_{0}\right)=-\frac{i}{2 N} \gamma^{\frac{2-z}{z}}\left\{\int_{0}^{\infty} \frac{d y}{\pi} \frac{y}{y^{z}+1}\right\}\left\{\int_{-1}^{0} \frac{d y}{2 \pi}|y|^{\frac{2-z}{z}}\right\} \operatorname{sgn}\left(k_{0}\right)\left|k_{0}\right|^{\frac{2}{z}} \frac{\lambda}{\sqrt{\lambda^{2}+\chi^{2}}} \\
& \Phi\left(k_{0}\right)=-\frac{i}{2 N} \gamma^{\frac{2-z}{z}}\left\{\int_{0}^{\infty} \frac{d y}{\pi} \frac{y}{y^{z}+1}\right\}\left\{\int_{-1}^{0} \frac{d y}{2 \pi}|y|^{\frac{2-z}{z}}\right\} \operatorname{sgn}\left(k_{0}\right)\left|k_{0}\right|^{\frac{2}{z}} \frac{\chi}{\sqrt{\lambda^{2}+\chi^{2}}}
\end{aligned}
$$

Equating this with Eq. (27), we obtain

$$
\sqrt{\lambda^{2}+\chi^{2}}=\frac{\gamma^{\frac{2-z}{z}}}{2}\left\{\int_{0}^{\infty} \frac{d y}{\pi} \frac{y}{y^{z}+1}\right\}\left\{\int_{-1}^{0} \frac{d y}{2 \pi}|y|^{\frac{2-z}{z}}\right\},
$$

where $\lambda$ and $\chi$ cannot be determined, but only $\lambda^{2}+\chi^{2}$ is given by Eq. (28). This is the trace of the $\Sigma\left(k_{0}\right) \longleftrightarrow$ $\Phi\left(k_{0}\right)$ symmetry in Eq. (26).

Appearance of the off diagonal self-energy is far from triviality. If the self-consistent equation for the anomalous part is linearized, one obtains the matrix equation

$$
\boldsymbol{A}_{k_{0}}=\boldsymbol{M}_{k_{0} k_{0}^{\prime}} \boldsymbol{A}_{k_{0}^{\prime}}
$$

where $\boldsymbol{A}_{k_{0}}$ corresponds to the pairing self-energy and the column index is frequency from zero to cutoff. This homogeneous equation does not have a nontrivial solution generally except the case when the matrix $\boldsymbol{M}_{k_{0} k_{0}^{\prime}}$ satisfies the particular condition of $\operatorname{Det}(\boldsymbol{M}-p \boldsymbol{I})=0$, where $p$ is the eigen value set to be 1 . Such a condition can be satisfied only at $T \leq T_{c}$ in the case of superconductivity, and the corresponding eigen vector $\boldsymbol{A}_{k_{0}}$ vanishes identically when $T>T_{c}$. The present situation is quite analogous with the $T=T_{c}$ case, i.e., the critical point, where $\boldsymbol{A}_{k_{0} \rightarrow 0} \rightarrow 0$ but $\boldsymbol{A}_{k_{0} \neq 0} \neq 0$. The off diagonal selfenergy correction may be regarded as one characteristic feature of the spin liquid state with a Fermi surface.

An interesting result from the anomalous self-energy is that the chiral symmetry $\boldsymbol{U}=\exp \left(i \theta \boldsymbol{\tau}_{3}\right)$ is broken at nonzero frequencies, indicating critical particle-hole composites with $2 k_{F}$. Figure 6 shows an imaginary part of the anomalous propagator in Eq. (24). As frequency increases, it is enhanced to a certain frequency and decreased, following the self-energy behavior. The maximum point becomes larger and its width gets broadened as the momentum $k_{x}$ of fermions increases. Since the anomalous pairing self-energy is identical to the normal self-energy, the normal Green's function displays qualitatively the same feature as the anomalous propagator. Appearance of critical particle-hole composites with $2 k_{F}$ is a novel feature of the two dimensional U(1) gauge theory with a Fermi surface.

\section{E. Thermodynamics}

We find physical implication of the present study from thermodynamics. Thermodynamics can be investigated in the Luttinger-Ward functional approach $\underline{16}$

$$
\begin{aligned}
& F\left[\boldsymbol{\Sigma}\left(k_{0}\right), \Pi\left(q_{0}, q\right)\right]=-N T \sum_{k_{0}} \int \frac{d^{2} k}{(2 \pi)^{2}} \boldsymbol{t r}\left\{\ln \left(\boldsymbol{\tau}_{\mathbf{3}} \boldsymbol{G}^{-1}\left(k_{0}, k\right)\right)-\boldsymbol{\Sigma}\left(k_{0}\right) \boldsymbol{\tau}_{\mathbf{3}} \boldsymbol{G}\left(k_{0}, k\right)\right\} \\
& +T \sum_{q_{0}} \int \frac{d^{2} q}{(2 \pi)^{2}}\left\{\ln \left(D^{-1}\left(q_{0}, q\right)\right)-\Pi\left(q_{0}, q\right) D\left(q_{0}, q\right)\right\} \\
& +T \sum_{k_{0}} T \sum_{q_{0}} \int \frac{d^{2} k}{(2 \pi)^{2}} \int \frac{d^{2} q}{(2 \pi)^{2}} \frac{1}{2} \boldsymbol{t r}\left\{\boldsymbol{\tau}_{\mathbf{3}} \boldsymbol{G}\left(k_{0}+q_{0}, k+q\right) D\left(q_{0}, q\right) \boldsymbol{\tau}_{\mathbf{3}} \boldsymbol{G}\left(k_{0}, k\right)\right\},
\end{aligned}
$$

where the last term is called the Luttinger-Ward functional, resulting from the Nambu-Eliashberg approximation in this paper.
Using the Nambu-Eliashberg equation, one can simplify this expression and obtain the following free energy 


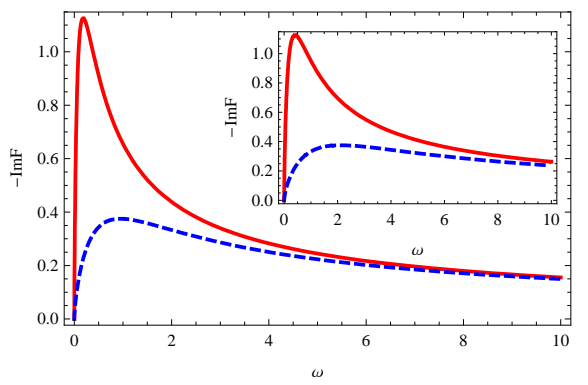

FIG. 6: (Color online) An imaginary part of the anomalous propagator in Eq. (24). The thick (red) line corresponds to $k_{x}=0.1$ and $k_{y}=0.0$ while the dashed (blue) line does to $k_{x}=0.3$ and $k_{y}=0.0$. The Landau damping coefficient is $\gamma=0.1$. Increasing the damping coefficient $\gamma$ from 0.1 to 0.5 , the hump becomes broadened slightly as shown in the inset figure.

$$
\begin{aligned}
& F(T)=-T \sum_{k_{0}} \int_{-\infty}^{\infty} \frac{d k_{y}}{2 \pi} \int_{-\infty}^{\infty} \frac{d k_{x}}{2 \pi} \ln \left\{\left(k_{y}^{2}+i \frac{\lambda}{N} \operatorname{sgn}\left(k_{0}\right)\left|k_{0}\right|^{\frac{2}{z}}\right)^{2}-k_{x}^{2}-\frac{\chi^{2}}{N^{2}}\left|k_{0}\right|^{\frac{4}{z}}\right\} \\
& +\frac{\Lambda^{2}}{2 \pi} T \sum_{q_{0}} \int_{-\infty}^{\infty} \frac{d q_{y}}{2 \pi}\left\{\ln \left(q_{y}^{z-1}+\gamma \frac{\left|q_{0}\right|}{\left|q_{y}\right|}\right)-\frac{\gamma \frac{\left|q_{0}\right|}{\left|q_{y}\right|}}{q_{y}^{z-1}+\gamma \frac{\left|q_{0}\right|}{\left|q_{y}\right|}}\right\}
\end{aligned}
$$

where $\Lambda$ is a momentum cutoff. It is trivial to observe that this free energy is qualitatively the same as that

$$
\begin{aligned}
& F(T)=-T \sum_{k_{0}} \int_{-\infty}^{\infty} \frac{d k_{y}}{2 \pi} \int_{-\infty}^{\infty} \frac{d k_{x}}{2 \pi} \ln \left\{\left(k_{y}^{2}+i \frac{\lambda}{N} \operatorname{sgn}\left(k_{0}\right)\left|k_{0}\right|^{\frac{2}{z}}\right)^{2}-k_{x}^{2}\right\} \\
& +\frac{\Lambda^{2}}{2 \pi} T \sum_{q_{0}} \int_{-\infty}^{\infty} \frac{d q_{y}}{2 \pi}\left\{\ln \left(q_{y}^{z-1}+\gamma \frac{\left|q_{0}\right|}{\left|q_{y}\right|}\right)-\frac{\gamma \frac{\left|q_{0}\right|}{\left|q_{y}\right|}}{q_{y}^{z-1}+\gamma \frac{\left|q_{0}\right|}{\left|q_{y}\right|}}\right\},
\end{aligned}
$$

except the anomalous self-energy part. In this respect thermodynamics of the Nambu-Eliashberg theory is essentially the same as that of the Eliashberg theory.

To perform integrals in Eq. (30) is straightforward. The boson sector gives rise to the conventional $T^{2}$ contribution while the fermion part results in the typical scaling expression $F(T) \propto T^{(d+z) / z}$ with $z=3$ and $d=2$ in the low temperature limit 17,18 .

An essential point in this demonstration is that thermodynamics is determined by the $z=3$ quantum criticality. This doe not seem to be consistent with the previous perturbative analysis $\frac{6}{-}$ although this study did not examine the thermodynamic potential in their perturbative scheme, thus a direct comparison with the present result is not possible. To construct the Luttinger-Ward functional consistent with this perturbative scheme is another problem. But, we expect that the singular part of the thermodynamic potential will be given by

$$
F_{s}(T) \propto-\frac{1}{\beta} \sum_{k_{0}} \int \frac{d^{2} \boldsymbol{k}}{(2 \pi)^{2}} \ln \left[-G_{f}^{-1}\left(i k_{0}, \boldsymbol{k}\right)\right]
$$

where $G_{f}\left(i k_{0}, \boldsymbol{k}\right)$ is the full fermion propagator with the AL-type vertex corrected self-energy. Since the fermion self-energy has an anomalous exponent, the specific heat coefficient will follow scaling different from $z=3$.

One may claim that this difference is natural because the Luttinger-Ward functional Eq. (30) does not seem to include the AL-type vertex corrections. However, this is not true. The key point in the present analysis is 
that the AL-type vertex corrections are not only introduced but also summed up to an infinite order in the Nambu-Eliashberg framework, taking into account the anomalous pairing self-energy. As a result, the anomalous exponent in the fermion self-energy disappears and thermodynamics remains qualitatively the same as that of the simple Eliashberg theory because the pairing selfenergy is essentially the same as the normal self-energy consistent with the $z=3$ scaling.

It should be pointed out that although we argued that the singular contribution for such vertex corrections will be kept within this anomalous pairing self-energy at least, we could not prove the equivalence exactly and showed it approximately as discussed in section II-B. If this "equivalence" turns out to be true, our statement has important physical meaning. In this respect it will be valuable to perform a direct summation for the AL-type vertex corrections, expected to confirm our result.

\section{DISCUSSION AND PERSPECTIVES}

\section{A. Discussion and summary}

Our study was motivated from a recent perturbative analysis ${ }^{6}$, based on the Eliashberg theory as the zeroth order, where the Aslamasov-Larkin (AL) -type vertex corrections give rise to an anomalous novel exponent for the fermion self-energy while they do not modify the Landau-damping boson self-energy. If this is a general feature beyond this level of approximation, various quantum critical phenomena ${ }^{1}$ should be reconsidered because the emergence of the anomalous exponent is expected to result in various novel critical exponents for thermodynamics, transport, and etc. In this respect it was crucial to investigate the existence of the anomalous exponent beyond this level of approximation.

Unfortunately, it was not easy to perform a direct nonperturbative summation for the AL-type vertex corrections due to retardation effects of gauge fluctuations although one can construct self-consistent equations analogous to superconducting instability. Instead, hinted from superconductivity (section II-B), we reformulated the non-perturbative summation, introducing an anomalous self-energy in the Nambu-spinor formalism, where the two components are momentum $k_{F}$ and $-k_{F}$ fermions, respectively. As a result, we found that the anomalous exponent of the normal self-energy turns out to disappear and the pairing self-energy has essentially the same functional form as the conventional Eliashberg self-energy for frequency. We argued that this is the result from the non-perturbative summation for the AL-type vertex cor- rections in a "unitary" transformed basis (Nambu-spinor formalism).

One implication of this result could be found in thermodynamics. If one evaluates the thermodynamic potential in a perturbative way with the AL-type vertex corrections, the appearance of the anomalous exponent in the fermion self-energy may give rise to a novel exponent for temperature dependence in the specific heat coefficient, different from the $z=3$ scaling result. Here, $z$ is the dynamic critical exponent. However, we argued that the non-perturbative summation for the AL-type vertex corrections, performed in the Nambu-Eliashberg framework, leaves the specific heat coefficient unchanged from the conventional Eliashberg theory with $z=3$. This is important for physical application to the heavy fermion quantum critical point, particularly, for the Kondo breakdown quantum critical point scenario $\frac{19}{19}$, where the $z=3$ quantum criticality gives a qualitatively good description for thermodynamics in a certain heavy fermion material 17 .

More direct implication of the anomalous self-energy may be found in spectroscopy measurements. For example, if the $2 k_{F}$ susceptibility (spin or charge) is measured (or evaluated), we expect that the anomalous propagator contributes and this will show unexpected composite states from critical fermions $\left(k_{F}\right.$ and $\left.-k_{F}\right)$ directly. Its detailed shape, for example, the peak or hump-like position and the width (life time of the state), is not clear at present. An optical conductivity (in the case of nematic quantum criticality) will also show an interesting feature. As expected, it would be enhanced at finite frequencies due to breaking of such preformed particle-hole pairs.

We also discussed the role of $2 k_{F}$ ladder vertex corrections in the critical state described by the simple Eliashberg theory. In particular, the issue was to prove existence of such a critical state as shows power law divergence of the $2 k_{F}$ spin susceptibility while the fermion selfenergy vanishes with a nontrivial exponent. Although this physics is typical at quantum criticality, we argued that this issue should be addressed more sincerely in the critical spin liquid state, i.e., self-consistently even in the relativistic $\mathrm{U}(1)$ gauge theory, where the $1 / N$ expansion is well defined.

\section{B. Perspectives}

Although the Nambu-Eliashberg theory does not give an anomalous exponent beyond the Eliashberg approximation, one can extend this framework, introducing the ladder-type vertex correction 


$$
\begin{aligned}
& \Pi\left(q_{0}, q\right)=\boldsymbol{t} \boldsymbol{r} \int \frac{d k_{0}}{2 \pi} \int \frac{d^{2} k}{(2 \pi)^{2}} \boldsymbol{\Lambda}\left(k_{0}+q_{0}, k+q ; k_{0}, k\right) \boldsymbol{\tau}_{\mathbf{3}} \boldsymbol{G}\left(k_{0}+q_{0}, k+q\right) \boldsymbol{\tau}_{\mathbf{3}} \boldsymbol{G}\left(k_{0}, k\right), \\
& \boldsymbol{\Sigma}\left(k_{0}\right)=-\frac{1}{N} \int \frac{d q_{0}}{2 \pi} \int \frac{d^{2} q}{(2 \pi)^{2}} \boldsymbol{\Lambda}\left(k_{0}+q_{0}, k+q ; k_{0}, k\right) \boldsymbol{\tau}_{\mathbf{3}} \boldsymbol{G}\left(k_{0}+q_{0}, k+q\right) D\left(q_{0}, q\right),
\end{aligned}
$$

where the two by two matrix vertex function is given by the ladder approximation

$$
\begin{aligned}
& \boldsymbol{\Lambda}\left(k_{0}+q_{0}, k+q ; k_{0}, k\right)=\boldsymbol{I}-\int \frac{d l_{0}}{2 \pi} \int \frac{d^{2} l}{(2 \pi)^{2}} \boldsymbol{\Lambda}\left(k_{0}+q_{0}-l_{0}, k+q-l ; k_{0}-l_{0}, k-l\right) \\
& \boldsymbol{\tau}_{\mathbf{3}} \boldsymbol{G}_{\sigma}\left(k_{0}+q_{0}-l_{0}, k+q-l\right) \boldsymbol{\tau}_{\mathbf{3}} \boldsymbol{G}_{\sigma}\left(k_{0}-l_{0}, k-l\right) D\left(l_{0}, l\right) .
\end{aligned}
$$

This can be regarded as natural generalization of the one patch formulation, taking the Nambu representation to incorporate $2 k_{F}$ backscattering. An important point is that the vertex function satisfies the Ward identity automatically because the Nambu-Eliashberg approximation is conserving, i.e., constructed from the Luttinger-Ward functional approach. We expect that the same strategy as the one patch formulation can be applied to the Nambu-Eliashberg theory for the two patch construction, where the Ward identity will help us introduce such vertex corrections $\frac{10}{}$. Another advantage in this formalism is that the $2 k_{F}$ ladder vertex will be introduced naturally. It is an interesting future direction to solve these equations.
We would like to thank T. Takimoto and S.-S. Lee for helpful discussions. K.-S. Kim was supported by the National Research Foundation of Korea (NRF) grant funded by the Korea government (MEST) (No. 2010-0074542).

\section{Appendix A: Evaluation of the polarization function}

We prove that the Landau damping boson self-energy Eq. (25) is preserved in the Nambu-Eliashberg theory. Inserting both normal and abnormal fermion Green's functions into the equation of the boson self-energy, we obtain

$$
\begin{aligned}
& \Pi\left(q_{0}, q\right)=2 \int_{-q_{0}}^{0} \frac{d k_{0}}{2 \pi} \int_{-\infty}^{\infty} \frac{d k_{y}}{2 \pi} \int_{-\infty}^{\infty} \frac{d k_{x}}{2 \pi} \\
& \frac{k_{x}\left(k_{x}+q_{x}\right)+k_{y}^{2}\left(k_{y}+q_{y}\right)^{2}-i \frac{\lambda}{N}\left|k_{0}\right|^{\frac{2}{z}} k_{y}^{2}+i \frac{\lambda}{N}\left|k_{0}+q_{0}\right|^{\frac{2}{z}}\left(k_{y}+q_{y}\right)^{2}+\frac{\lambda^{2}+\chi^{2}}{N^{2}}\left|k_{0}+q_{0}\right|^{\frac{2}{z}}\left|k_{0}\right|^{\frac{2}{z}}}{\left\{\left(k_{x}+q_{x}\right)^{2}-\left(k_{y}+q_{y}\right)^{4}-2 i \frac{\lambda}{N}\left|k_{0}+q_{0}\right|^{\frac{2}{z}}\left(k_{y}+q_{y}\right)^{2}+\frac{\lambda^{2}+\chi^{2}}{N^{2}}\left|k_{0}+q_{0}\right|^{\frac{4}{z}}\right\}\left\{k_{x}^{2}-k_{y}^{4}+2 i \frac{\lambda}{N}\left|k_{0}\right|^{\frac{2}{z}} k_{y}^{2}+\frac{\lambda^{2}+\chi^{2}}{N^{2}}\left|k_{0}\right|^{\frac{4}{z}}\right\}},
\end{aligned}
$$

where both normal and pairing self-energies are explicitly used.
It is straightforward to perform the integration of $k_{x}$, resulting in

$$
\Pi\left(q_{0}, q\right)=\frac{1}{2 \pi} \int_{-q_{0}}^{0} \frac{d k_{0}}{2 \pi} \int_{-\infty}^{\infty} \frac{d k_{y}}{2 \pi} \frac{\sqrt{D}\left((B+C)(C-D)+(B-C) q_{x}^{2}\right)+\sqrt{C}\left((B+D)(C-D)-(B-D) q_{x}^{2}\right)}{\sqrt{C} \sqrt{D}\left((C-D)^{2}-2(C+D) q_{x}^{2}+q_{x}^{4}\right)},
$$


where

$$
\begin{aligned}
B & =k_{y}^{2}\left(k_{y}+q_{y}\right)^{2}-i \frac{\lambda}{N}\left|k_{0}\right|^{\frac{2}{z}} k_{y}^{2}+i \frac{\lambda}{N}\left|k_{0}+q_{0}\right|^{\frac{2}{z}}\left(k_{y}+q_{y}\right)^{2}+\frac{\lambda^{2}+\chi^{2}}{N^{2}}\left|k_{0}+q_{0}\right|^{\frac{2}{z}}\left|k_{0}\right|^{\frac{2}{z}}, \\
C & =\left(k_{y}+q_{y}\right)^{4}+2 i \frac{\lambda}{N}\left|k_{0}+q_{0}\right|^{\frac{2}{z}}\left(k_{y}+q_{y}\right)^{2}-\frac{\lambda^{2}+\chi^{2}}{N^{2}}\left|k_{0}+q_{0}\right|^{\frac{4}{z}}, \\
D & =k_{y}^{4}-2 i \frac{\lambda}{N}\left|k_{0}\right|^{\frac{2}{z}} k_{y}^{2}-\frac{\lambda^{2}+\chi^{2}}{N^{2}}\left|k_{0}\right|^{\frac{4}{z}} .
\end{aligned}
$$

Scaling $k_{y}$ and $k_{0}$ as follows

$$
k_{y}=q_{y} y, \quad k_{0}=q_{0} t,
$$

the above expression reads

$$
\Pi\left(q_{0}, q\right)=\frac{1}{2 \pi} \frac{\left|q_{0}\right|}{\left|q_{y}\right|} \int_{-1}^{0} \frac{d t}{2 \pi} \int_{-\infty}^{\infty} \frac{d y}{2 \pi} \frac{\sqrt{d}\left((b+c)(c-d)+(b-c) \frac{q_{x}^{2}}{q_{y}^{2}}\right)+\sqrt{c}\left((b+d)(c-d)-(b-d) \frac{q_{x}^{2}}{q_{y}^{2}}\right)}{\sqrt{c} \sqrt{d}\left((c-d)^{2}-2(c+d) \frac{q_{x}^{2}}{q_{y}^{2}}+\frac{q_{x}^{4}}{q_{y}^{4}}\right)},
$$

where $b, c$, and $d$ are given by

$$
\begin{aligned}
& B=q_{y}^{4}\left\{y^{2}(y+1)^{2}-i \frac{\lambda}{N}|t|^{\frac{2}{z}} y^{2}\left(\frac{\left|q_{0}\right|^{\frac{1}{z}}}{\left|q_{y}\right|}\right)^{2}+i \frac{\lambda}{N}|t+1|^{\frac{2}{z}}(y+1)^{2}\left(\frac{\left|q_{0}\right|^{\frac{1}{z}}}{\left|q_{y}\right|}\right)^{2}+\frac{\lambda^{2}+\chi^{2}}{N^{2}}|t+1|^{\frac{2}{z}}|t|^{\frac{2}{z}}\left(\frac{\left|q_{0}\right|^{\frac{1}{z}}}{\left|q_{y}\right|}\right)^{4}\right\} \equiv q_{y}^{4} b \\
& C=q_{y}^{4}\left\{(y+1)^{4}+2 i \frac{\lambda}{N}|t+1|^{\frac{2}{z}}(y+1)^{2}\left(\frac{\left|q_{0}\right|^{\frac{1}{z}}}{\left|q_{y}\right|}\right)^{2}-\frac{\lambda^{2}+\chi^{2}}{N^{2}}|t+1|^{\frac{4}{z}}\left(\frac{\left|q_{0}\right|^{\frac{1}{z}}}{\left|q_{y}\right|}\right)^{4}\right\} \equiv q_{y}^{4} c \\
& D=q_{y}^{4}\left\{y^{4}-2 i \frac{\lambda}{N}|t|^{\frac{2}{z}} y^{2}\left(\frac{\left|q_{0}\right|^{\frac{1}{z}}}{\left|q_{y}\right|}\right)^{2}-\frac{\lambda^{2}+\chi^{2}}{N^{2}}|t|^{\frac{4}{z}}\left(\frac{\left|q_{0}\right|^{\frac{1}{z}}}{\left|q_{y}\right|}\right)^{4}\right\} \equiv q_{y}^{4} d
\end{aligned}
$$

An important point is that this integral is convergent near $y \approx 0$. As a result, the main contribution comes from

$$
|y| \gg 1
$$

Scaling $y$ in the following way

$$
Y=y\left(\frac{\left|q_{0}\right|^{\frac{1}{z}}}{\left|q_{y}\right|}\right)^{-1},
$$

we obtain

$$
c-d \approx 4 Y^{3}, \quad b-c \approx-2 Y^{3}, \quad b-d \approx 2 Y^{3}
$$

in the dominant region for the integral. Finally, we find the boson self-energy

$$
\begin{aligned}
& \Pi\left(q_{0}, q\right) \approx \frac{1}{\pi} \frac{\left|q_{0}\right|}{\left|q_{y}\right|} \int_{-1}^{0} \frac{d t}{2 \pi} \int_{\left(\frac{\left|q_{0}\right|^{\frac{1}{z}}}{q_{y} \mid}\right)^{-1}}^{\infty} \frac{d Y}{2 \pi} \frac{Y^{2}\left\{4 Y^{7}-2 Y^{3} \frac{q_{x}^{2}}{q_{y}^{2}}\left(\frac{\left|q_{0}\right|^{\frac{1}{z}}}{\left|q_{y}\right|}\right)^{-4}\right\}}{Y^{4}\left\{16 Y^{6}-4 Y^{4} \frac{q_{x}^{2}}{q_{y}^{2}}\left(\frac{\left|q_{0}\right|^{\frac{1}{z}}}{\left|q_{y}\right|}\right)^{-2}+\frac{q_{x}^{4}}{q_{y}^{4}}\left(\frac{\left|q_{0}\right|^{\frac{1}{z}}}{\left|q_{y}\right|}\right)^{-6}\right\}} \\
& \approx \frac{1}{\pi} \frac{\left|q_{0}\right|}{\left|q_{y}\right|} \int_{-1}^{0} \frac{d t}{2 \pi} \int_{\left(\frac{\left|q_{0}\right|^{\frac{1}{z}}}{\left|q_{y}\right|}\right)^{-1}}^{\Lambda} \frac{d Y}{2 \pi} \frac{1}{4 Y}=\frac{1}{16 \pi^{3}} \frac{\left|q_{0}\right|}{\left|q_{y}\right|}\left\{\ln \Lambda+\ln \left(\frac{\left|q_{0}\right|^{\frac{1}{z}}}{\left|q_{y}\right|}\right)\right\} \approx \frac{\ln \Lambda}{16 \pi^{3}} \frac{\left|q_{0}\right|}{\left|q_{y}\right|},
\end{aligned}
$$

nothing but the Landau damping form. In this respect Eq. (25) and Eq. (27) are the fully self-consistent solu- tion of the Nambu-Eliashberg equations. 
1 P. Gegenwart, Q. Si, and F. Steglich, Nature Physics 4, 186 (2008); H. v. Lohneysen, A. Rosch, M. Vojta, and P. Wolfle, Rev. Mod. Phys. 79, 1015 (2007).

2 P. A. Lee, N. Nagaosa, and X.-G. Wen, Rev. Mod. Phys. 78, 17 (2006).

3 T. Senthil, A. Vishwanath, L. Balents, S. Sachdev, and M. P. A. Fisher, Science 303, 1490 (2004); T. Senthil, L. Balents, S. Sachdev, A. Vishwanath, and M. P.A. Fisher, Phys. Rev. B 70, 144407 (2004).

4 T. Moriya and J. Kawabata, J. Phys. Soc. Jpn. 34, 639 (1973); T. Moriya and J. Kawabata, J. Phys. Soc. Jpn. 35, 669 (1973); J. A. Hertz, Phys. Rev. B 14, 1165 (1976); A. J. Millis, Phys. Rev. B 48, 7183 (1993).

${ }^{5}$ Sung-Sik Lee, Phys. Rev. B 80, 165102 (2009).

${ }^{6}$ Max A. Metlitski and S. Sachdev, arXiv:1001.1153 (unpublished).

7 Max A. Metlitski and S. Sachdev, arXiv:1005.1288 (unpublished).

8 David F. Mross, J. McGreevy, H. Liu, and T. Senthil, Phys. Rev. B 82, 045121 (2010).

9 D. L. Maslov, arXiv:cond-mat/0506035 (Lecture notes for the LXXXI Les Houches Summer School "Nanoscopic Quantum Transport", 2004).

10 Ki-Seok Kim, arXiv:1004.3442, to be published in Phys. Rev. B.

11 J. R. Schrieffer, Theory of Superconductivity (Westview Press, Colorado, 1999).

12 J. Rech, C. Pepin, and A. V. Chubukov, Phys. Rev. B 74, 195126 (2006).
13 B. L. Altshuler, L. B. Ioffe, and A. J. Millis, Phys. Rev. B. 50, 14048 (1994).

14 W. Rantner and X.-G. Wen, Phys. Rev. B 66, 144501 (2002).

15 D. H. Kim and P. A. Lee, Annals Phys. 272, 130 (1999).

16 J. M. Luttinger and J. C. Ward, Phys. Rev. 118, 1417 (1960); G. Baym and L. P. Kadanoff, Phys. Rev. 124, 287 (1961).

17 K.-S. Kim, A. Benlagra, and C. Pépin, Phys. Rev. Lett. 101, 246403 (2008).

18 This $z=3$ scaling property can be understood from the total self-energy in the normal Green's function, given by

$$
\Sigma_{t o t}^{s}\left(k_{0}, k\right)=\frac{\left|\Phi\left(k_{0}\right)\right|^{2}}{i \eta k_{0}-s k_{x}+k_{y}^{2}-\Sigma\left(k_{0}\right)} .
$$

In the long wave length limit this total self-energy can be approximated as follows

$$
\Sigma_{\text {tot }}^{s}\left(k_{0}, k\right) \approx-\frac{\left|\Phi\left(k_{0}\right)\right|^{2}}{\Sigma\left(k_{0}\right)}=-i \frac{\chi^{2} / \lambda}{N} \operatorname{sgn}\left(k_{0}\right)\left|k_{0}\right|^{\frac{2}{z}} .
$$

This expression is essentially the same as the conventional Eliashberg self-energy. In this respect we expect that low energy physics does not change from the Eliashberg dynamics.

19 I. Paul, C. Pepin, and M. R. Norman, Phys. Rev. Lett. 98, 026402 (2007); C. Pepin, Phys. Rev. Lett. 98, 206401 (2007). 